\title{
Investigation and Research on College Students' social media dependence
}

\author{
Haiyan Liu ${ }^{\text {a }}$, Jingju Hou ${ }^{\text {b }}$ \\ Information Technology Institute, Jilin Agricultural University, Changchun 130117, China \\ ahaiyan_49@sohu.com, bzzjj18zz@sina.com
}

\begin{abstract}
This paper the college students in Changchun area as the research object, such as weibo, WeChat, QQ instant social network platform, for example, using the method of literature, questionnaire, interview method and statistical analysis, this paper discusses the college students' social media usage and rely on analysis of the social media depends on the consequences and the corresponding solutions. In order to through the discussion on the problem, can college educational administration managers to better students' usage of social media to correctly guide.
\end{abstract}

Keywords: College students; Social media dependence; Social platform.

\section{Introduction}

As the network becomes more and more perfect, and constantly update the intelligent mobile equipment upgrading, social media become indispensable communication and entertainment tool, social media people can write, evaluation, discussion, sharing and mutual communication. Social media to meet people's communication, access to information, the various needs of daily entertainment. The more a person depends on the media to meet their needs, the role of media in this person's life is more important, the more a person depends on the media, and the media of the people's influence is greater. College students as the main use of social media groups, the use of social media, how to use the situation, the performance of social media depends on what? What effects will be produced? In this paper, 383 college students from six universities, Jilin Agricultural University, Northeast Normal University, Changchun University of Traditional Chinese Medicine, Jilin University of architecture, Northeast Normal University, College of Humanities and the Jilin University were tested for statistical analysis of the questionnaire results and conclusions. Firstly, it analyzes the characteristics and performance of social media, and then analyzes the impact of social media on college students, and finally puts forward the corresponding solutions.

\section{Survey on the use of social media in College Students}

In this survey, the proportion of male and female was equal, and the male and female were $50.13 \%$ and $49.87 \%$ respectively. Among them, $31.07 \%$ share of $31.07 \%$ freshman and sophomore, junior year accounted for $29.5 \%$, accounting for $6.79 \%$ of all senior graduate students accounted for $4.18 \%$. According to the survey found that the current college students use frequency in the top three social platforms, respectively is: WeChat, QQ, weibo, its using ratio of $83.07 \%, 80.47 \%$ and $49.74 \%$ respectively. Can see $\mathrm{WeChat}$ is the highest immediate social platform, in the current college students use QQ use 2.6\%, lower than the WeChat and weibo use compared with the former two nearly half of the less quantity. At the same time, there are a few students use in addition to social media, such as Facebook, twitter, etc.

\subsection{Social media use purposes.}

Subjects according to the purposes of use frequency sorting, option average composite scores, summing up the eight kinds of purposes of use frequency, average comprehensive scoring option $=(\Sigma$ frequency by weight)/subjects, it reflects the comprehensive ranking of options, the higher the score according to comprehensive ordering more.

As shown in Figure 1, the use of social media use, with relatives and friends to chat with the highest average score, while understanding the dynamics of others as well as the release of dynamic frequency is relatively high. Transfer files, shopping, learning, entertainment, gossip, and 
understanding of the use of current news and other uses frequency decreased in turn, there are still some subjects use social media have other purposes.

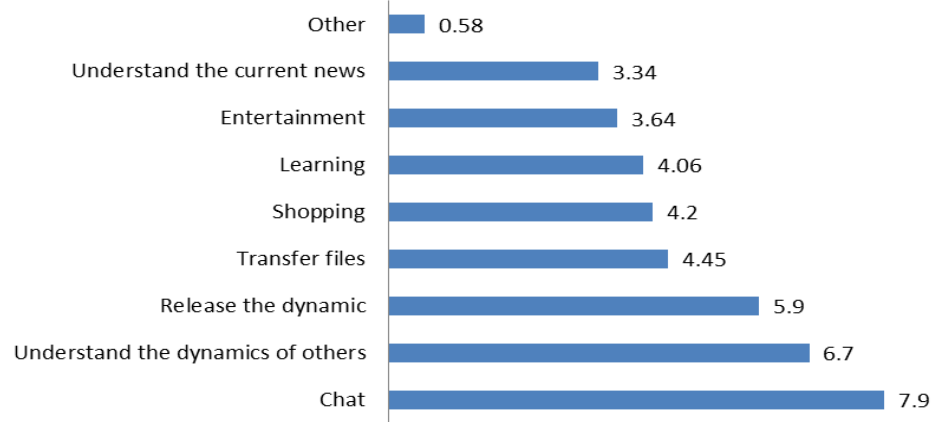

Fig. 1 Schedule for social media usage purposes

\subsection{Social media use frequency and duration of use}

Survey subjects per hour and the frequency of the use of social media, with five times at intervals, setting options 0 to 5, 6-10,11-15 and above 15 times, respectively in social media "basic does not rely on", "mild dependence" and "moderate" and "heavy reliance on". At the same time, the investigation subjects using social media time every day, in hours, respectively, setting options within 1 hour, 1 to 2 hours, 2-3 hours and 3 hours to investigate four interval. The results of the survey are shown in figure 2 and figure 3.
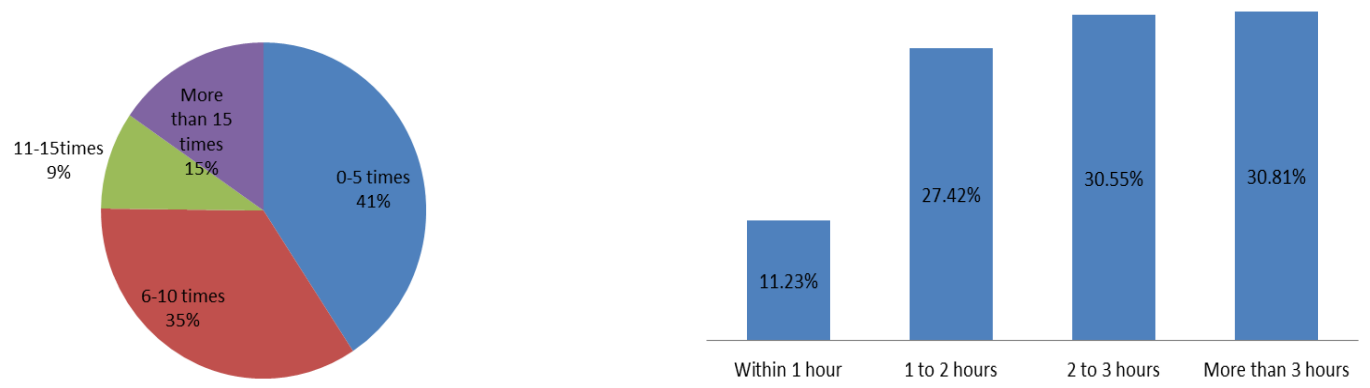

Fig. 2 Social media use frequency statistics Fig.3 Social media use a long statistical chart every day

\subsection{Survey of College Students' social media dependence}

For the specific embodiment of social media dependence, this article on the study of social media groups of college students to set seven questions, the answer is set to often, occasionally, rarely, never four levels. The answer is "often" subjects as severe social media dependence; the answer is "occasionally" subjects as moderate dependence; the answer is "a few" subjects as mild dependence; the answer is "never" subjects as basic does not depend on. Statistical results are shown in Table 1.

As shown in Table 1, 87.39\% of the participants have continued to refresh the social media and look forward to the new message; only $2 \%$ of the subjects have never been held on this topic. The use of social media time has exceeded expectations of subjects as high as $82.43 \% .88 .25 \%$ subjects with anxiety and other emotions in not using social media, $82.51 \%$ of the subjects had different degrees of feeling using social media can't be met but difficult to stop, $88.25 \%$ subjects are trying different degree but did not get obvious effect to reduce the use of social media phenomenon. Subjects who were unable to concentrate on their studies or focused on the social media were $90.6 \%$ of all subjects. $89.56 \%$ of subjects cut their study or work in varying degrees by using social media. According to the results of the survey can be further explained by the participants in the social media dependence and dependence. The participants' dependence on social media is reflected in their behavior is difficult to control, negative emotions, can't be met and try to reduce the use of no fruit, and so on. For the above phenomenon, the subjects' degree of social media has mild dependence and different degrees of moderate and severe dependence on social media, and a relatively high degree of dependence of subjects (moderate and severe dependence) the number is more than the less dependent subjects (mild dependence and little dependence). The number of as can be seen, the degree of dependence on social media in college students is quite serious. 
Table 1 Statistical table of College Students' social media dependence

\begin{tabular}{cccccc}
\hline \multirow{2}{*}{ Number } & Evaluating & \multicolumn{4}{c}{ Answer set } \\
& indicator & Often & Occasionally & Few times & Never \\
\hline 1 & Constantly refresh & $30.03 \%$ & $48.04 \%$ & $19.32 \%$ & $2.61 \%$ \\
2 & Time can't control & $30.92 \%$ & $43.73 \%$ & $21.41 \%$ & $7.57 \%$ \\
3 & Interrupt the original plan & 21.41 & $42.04 \%$ & $26.11 \%$ & $10.44 \%$ \\
4 & Self-control & $12.79 \%$ & $32.38 \%$ & $37.34 \%$ & $17.49 \%$ \\
5 & Try to use less time has no significant effect & $11.75 \%$ & $34.99 \%$ & $36.55 \%$ & $16.71 \%$ \\
6 & Restless when not in use & $27.68 \%$ & $36.55 \%$ & $26.37 \%$ & $9.4 \%$ \\
\hline
\end{tabular}

\section{Analysis on the reasons of College Students' social media dependence}

First of all, social media's own characteristics meet the needs of College Students. Social media has changed the way people use the Internet, from the recipient of a huge amount of information to become a huge producer of information. Sharing, interaction between the circles of friends to narrow the distance between College students. Students enjoy this kind of communication. College students enjoy this way of communication. Social media and college students to coincide with the pace of life, the university learning environment relatively loose, and college students have strong social aspirations, they enjoy instant social network brings the relaxed and happy, that is the best arrangement of study and life.

Secondly, the impact of the surrounding environment, such as the celebrity effect of the drive. Micro-blog and other social media operator's flagship star effect of the promotion strategy of college students have achieved great success, worship and yearning for the stars, and they want to shorten the distance, inquire about their movements. Social media provides an excellent platform for.

Finally, College student generally have poor time management, weak self-control and learning difficulty concentrating in the problem, it is difficult to do things well, difficult to adhere to, give up easily, of course, these difficulties, and the social environment, family education background have a direct relationship.

\section{The impact of social media dependence}

The mobile phone has the information on social media openness and freedom, the relevant departments is difficult to play the role of gatekeeper, coupled with their own lack of knowledge, lack of guidance from all walks of life, they are easily addicted to social media groups to create a "Pseudo Environment" and even confusion network inextricably bogged down in, and the social reality social, cause interpersonal indifference, values and world view distortion and other adverse effects.

The virtual network community meeting seriously affects people's communication and communication skills. Interaction in the network will not only affect the reality of human interaction, but also affect their relationship. In addition, the excessive dependence of social media on College Students' physical and mental health has also caused some impact. Social media to create a pseudo environment so that they hide their true identity, not to reveal their true information, lost the basic moral cognition and evaluation. As part of the college students in order to make up the feelings of emptiness, picking up strange friends through social media, dissemination of pornographic pictures, and other nude video chat, and even some students will meet with each other improper relationship. If things go on like this, will seriously affect their physical and mental health. At the same time, frequent contact with mobile social media will make people become more eccentric personality, and adverse effects on vision and nervous system.

\section{The solution of the college students' social media dependence}

\subsection{To improve the relevant laws and regulations of social media}

The new social media is virtual, openness; therefore, need more relevant laws and regulations for social media development escort, through the relevant regulations, to promote the healthy 
development of the construction of social media platform. In addition, due to lack of experience, social impulsive characteristics of college students, college students should be strengthened to the social network construction regulations, actively promote the social networking tool backstage real name system, cultivate students' integrity awareness, improve the security of communication, enhance students' interpersonal skills, norms of social behavior of Internet users in the network media.

\subsection{Guide the behavior of college students in social media habits}

Colleges and universities should aim at the new characteristics of College Students' network communication under the new media environment, to carry out relevant management and education work, and to correct the social behavior of college students. If you can through the opening of interpersonal communication, network security and network regulations and other related courses, to guide the use of social media in college students, to encourage students to more into the reality of interpersonal communication.

\subsection{Strengthen the media literacy education of College Students}

Media literacy including information selection ability, query ability, comprehension and evaluation capacity, media literacy education can effectively promote the students' evaluation and management of individual media contact behavior, society, schools and families should be combined with students' media literacy education, including how to use the media, how to determine the value and significance of media information.

\section{Conclusion}

The higher the degree of dependence on social media, the greater the possibility of social life, the greater the possibility, it will inevitably lead to interpersonal barriers and barriers. Therefore, on the one hand the use of college students in the future media process, to grasp the use of long, to master the correct way of social communication; on the other hand, the school and the society can give certain media literacy education to improve college students' media habits, to avoid the disadvantages.

\section{Acknowledgments}

This work was financially supported by Jilin Agricultural University undergraduate science and Technology Innovation Fund, Project name: College students rely on social media and academic procrastination research.

\section{References}

[1] Guilan Che. The behavior of college students in the new social media habits and guides the strategy research. Journal of Cangzhou Normal University. Vol. 32 (2016) No. 2, p. 38-40.

[2] Gaofang Jiang; Zihao Yang; Rina Su; et al. Research on self-acceptance of college students and the relationship between self-acceptance and mobile social media. Journal of Cangzhou Normal University. Vol. 32 (2016) No. 1, p. 84-87.

[3] Zhensheng Liu. Research on social media dependence and media demand: a case study of College Students' micro-blog dependence. Journalism Bimonthly. Vol. 30 (2016) No. 4, p. 119-121. 